\title{
Comparação dos perfis dos pacientes hipertensos atendidos em Pronto-Socorro e em tratamento ambulatorial ${ }^{*}$
}

\author{
COMPARISON OF THE PROFILE OF HYPERTENSIVE PATIENTS SEEN IN EMERGENCY UNIT \\ WITH THOSE RECEIVING OUTPATIENT CLINIC TREATMENT
}

\begin{abstract}
PERFIL DE LOS PACIENTES HIPERTENSOS ATENDIDOS EN UN SERVICIO DE EMERGENCIA: COMPARACIÓN CON HIPERTENSOS EN TRATAMIENTO AMBULATORIO
\end{abstract}

\author{
Cristiane Garcia Sanchez ${ }^{1}$, Angela Maria Geraldo Pierin ${ }^{2}$, Décio Mion Jr. ${ }^{3}$
}

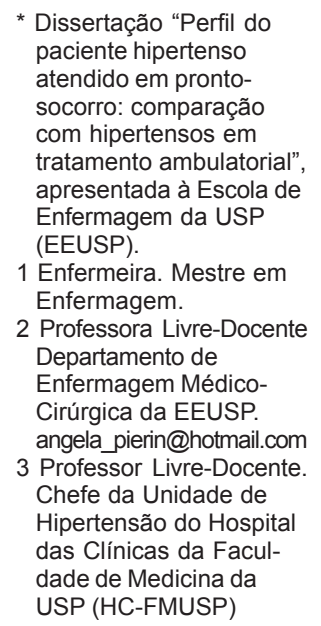

1 Enfermeira. Mestre em Enfermagem.

2 Professora Livre-Docente Departamento de Enfermagem MédicoCirúrgica da EEUSP. angela_pierin@hotmail.com

3 Professor Livre-Docente. Chefe da Unidade de Hipertensão do Hospital das Clínicas da Faculdade de Medicina da USP (HC-FMUSP)

\author{
RESUMO \\ O estudo comparou 100 \\ hipertensos atendidos no \\ Pronto-socorro com 100 \\ pacientes do Ambulatório. Os \\ hipertensos do Pronto- \\ Socorro foram diferentes \\ $(p<0,05)$ em relação $a$ : \\ maior pressão arterial; menor \\ renda salarial; maior \\ consumo de bebida alcoólica; \\ não pertenciam à comunidade \\ do hospital; descobriram ser \\ hipertensos por sentirem-se \\ mal; mediram menos a \\ pressão; e deixaram de tomar \\ mais medicamentos. A análise \\ multivariada revelou \\ diferenças significativas entre \\ os dois grupos quanto à \\ renda, ao local onde é medida \\ a pressão e não tomar os \\ medicamentos. Concluiu-se \\ que características \\ desfavoráveis podem \\ contribuir para não realizar o \\ tratamento anti-hipertensivo, \\ levando a atendimentos em \\ unidades de emergência.
}

\section{PALAVRAS-CHAVE}

Hipertensão.

Anti-hipertensivos (uso terapêutico).

Adesão.

Serviços médicos de emergência.

Assistência ambulatorial.

\begin{abstract}
This study compares 100 hypertensive patients in emergency units with those who were seen in outpatient clinics. Hypertensive patients seen at the emergency unit showed to be different $(p<0.05)$ from those seen at the outpatient clinic regarding: higher blood pressure; lower income; not belonging to the hospital community; greater alcohol intake; became aware of their hypertension because felt bad; measured blood pressure less often; did not take the medicine more often. In conclusion, unfavorable characteristics can contribute to hypertensive patients who do not follow anti-

hypertension treatment adequately, leading to care in emergency units.
\end{abstract}

\section{KEYWORDS}

Hypertension.

Anti-hypertension treatment. Compliance.

Emergency medical services. Ambulatory care.

\section{RESUMEN}

Este estudio comparó 100 hipertensos atendidos en un servicio de emergencia, con 100 pacientes de consulta externa. Los hipertensos de emergencia fueron diferentes $(p<0,05)$ en relación $a$ : presión arterial más elevada; menor renta salarial; no pertenecian a la comunidad del hospital; mayor consumo de bebidas alcohólicas; descubrieron ser hipertensos por sentirse mal; se midieron menos la presión y dejaron de tomar los medicamentos. El análisis multivariado muestra que la renta, el local donde se mide la presión y dejar de tomar los medicamentos marcaron diferencias entre los dos grupos. Se concluyó que estas características desfavorables pueden contribuir al abandono del tratamiento antihipertensivo llevando a los pacientes a ser atendidos en unidades de emergencia.

\section{PALABRAS CLAVE}

Hipertension.

Agentes antihipertensivos (uso terapêutico).

Adesão

Serviços médicos de emergencía.

Atencion ambulatoria 


\section{INTRODUÇÃO}

Um dos motivos mais freqüentes de admissão de pacientes hipertensos nos serviços de emergência é a elevação súbita da pressão arterial e, geralmente, quando a doença é descoberta. As situações de urgência ou emergência hipertensiva com comprometimento de órgãos nobres como o coração, rins e cérebro, podem acarretar seqüelas irreversíveis, ou levar o indivíduo à morte ${ }^{(1-5)}$.

A hipertensão arterial é considerada um problema de saúde pública pela elevada prevalência de $15 \%$ a $20 \%$ na população adulta, além de constituir importante fator de risco coronário. Relaciona-se a $40 \%$ dos óbitos por doenças cardiovasculares e a uma maior chance de desenvolver complicações como acidente vascular cerebral, infarto do miocárdio e insuficiência cardíaca ${ }^{(6-9)}$. Tratase de uma doença crônica, na maioria das vezes assintomática, e que requer tratamento para toda a vida para o seu controle.

Qualquer que seja o tratamento proposto é importante obter-se a adesão contínua do paciente às medidas recomendadas. Estudos relatam ser comum os pacientes hipertensos abandonarem o tratamento, uma vez tendo sua pressão arterial controlada, voltando então aos níveis pressóricos anteriores. A não adesão ao tratamento proposto tem atingido aproximadamente $50 \%$ dos pacientes ${ }^{(10-12)}$ A falta de controle da pressão arterial mesmo em pacientes seguidos regularmente é freqüente. Dados do National Health and Nutrition Examination Survey (NHANES III) ${ }^{(7)}$ mostram que, dentre os hipertensos nos Estados Unidos, 54\% estão sob tratamento e apenas $27 \%$ estão controlados. A falta de adesão ao tratamento pode ser atribuída a vários fatores, destacando-se os relaciona-dos ao pacientes, como hábitos de vida, crença, hábitos culturais; à doença, como a cronicidade e ausência de sintomas e ao tratamento, como efeitos indesejáveis das drogas e acesso ao tratamento.

O diagnóstico e o tratamento adequados proporcionam menores gastos com internações, invalidez, bem como com a assistência às cardiopatias, acidentes vasculares cerebrais e suas seqüelas, reduzindo também a procura aos serviços de emergência.

O fato que despertou a atenção para a realização do presente estudo foi a alta freqüência de pacientes hipertensos que chegavam ao setor de emergência de um hospital universitário com a pressão arterial descontrolada e quais seriam os motivos que levariam estes pacientes hipertensos a serem atendidos em situação de emergência.

Portanto, este trabalho teve como objetivo comparar pacientes hipertensos atendidos no Pronto-socorro com aqueles seguidos regularmente em Ambulatório quanto a: dados biossociais, hábitos de vida, conhecimento sobre a doença e tratamento $\mathrm{e}$ níveis da pressão arterial.

\section{CASUÍSTICA E MÉTODO}

Realizou-se estudo descritivo prospectivo no setor de Emergência e Ambulatório do Hospital Universitário da Universidade de São Paulo. Foram estudados 100 hipertensos atendidos no setor de emergência de adultos e 100 seguidos regularmente no ambulatório, de ambos os sexos, com idade mínima de 18 anos, conscientes, orientados e que concordaram em participar do estudo após assinarem o termo de conhecimento livre e esclarecido. O estudo foi aprovado pela Comissão de Ética da instituição. No ambulatório a amostra foi composta de forma pareada por idade e sexo em relação aos pacientes entrevistados no Pronto-Socorro.

Para a coleta de dados utilizou-se instrumento específico para entrevista, além de consulta à ficha de admissão dos pacientes do Pronto-socorro e o prontuário dos pacientes do Ambulatório.

Todos os dados foram processados no sistema Statistic Package for Social Sciencies versão 7.5. As variáveis classificatórias foram submetidas à análise univariada (teste QuiQuadrado e teste exato de Fisher). As médias foram comparadas com testes paramétricos e não paramétricos (teste t-Student, análise de variância, teste da soma de postos de Wilcoxon e Kruskal-Wallis). Para análise multivariada algumas variáveis foram obtidas através da análise de regressão logística, expressa em odds ratio-OR (razão de chances) e seus intervalos de confiança através da exponenciação dos coeficientes de regressão e erros-padrão. $O$ nível de significância adotado foi de 0,05 .

\section{RESULTADOS}

Os dados da Tabela 1 mostram que, entre as variáveis biossociais, apenas a renda e a
Comparação dos perfis dos pacientes hipertensos atendidos em Pronto-socorro e em tratamento ambulatorial 
Cristiane Garcia Sanchez Angela Maria G. Pierin Décio Mion Jr.

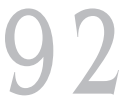

Rev Esc Enferm USP 2004; 38(1): 90-8. origem dos pacientes classificados em comunidade pertencente à Universidade de São Paulo e Butantã e comunidade não pertencente à Universidade de São Paulo e Butantã, influenciaram na comparação entre os hipertensos atendidos no Pronto-socorro e no Ambulatório. Os pacientes atendidos no
Pronto-socorro referiram renda significativamente menor $(<5$ salários-mínimos, $\mathrm{p}<0,05)$ em relação aos hipertensos do Ambulatório. Quanto à origem, 84\% dos pacientes do Pronto-socorro não pertenciam à comunidade USP e Butantã contra apenas $23 \%$ dos pacientes do Ambulatório $(\mathrm{p}<0,05)$.

Tabela 1 - Caracterização dos pacientes hipertensos atendidos no ProntoSocorro e Ambulatório segundo sexo, idade, raça, escolaridade, ocupação, renda,hábitos de vida e pressão arterial [admissão no Pronto-socorro e de três consultas ambulatoriais] (São Paulo, 2001)

\begin{tabular}{|c|c|c|c|c|c|c|}
\hline \multirow[t]{2}{*}{ Características } & \multicolumn{2}{|c|}{ Pronto-socorro } & \multicolumn{2}{|c|}{ Ambulatório } & \multicolumn{2}{|c|}{ Total } \\
\hline & $\mathbf{n}^{\mathbf{o}}$ & $\%$ & $\mathbf{n}^{\mathbf{o}}$ & $\%$ & $\mathbf{n}^{\mathbf{o}}$ & $\%$ \\
\hline \multicolumn{7}{|l|}{ Sexo } \\
\hline Feminino & 62 & 62 & 62 & 62 & 124 & 62 \\
\hline Masculino & 38 & 38 & 38 & 38 & 76 & 38 \\
\hline \multicolumn{7}{|l|}{ Idade } \\
\hline Anos (média + DP) & \multicolumn{2}{|c|}{$60+12$} & \multicolumn{2}{|c|}{$56+11$} & \multicolumn{2}{|c|}{$58+11$} \\
\hline \multicolumn{7}{|l|}{ Cor } \\
\hline Branca & 31 & 31 & 27 & 27 & 58 & 29 \\
\hline Não branca & 69 & 69 & 73 & 73 & 142 & 71 \\
\hline \multicolumn{7}{|l|}{ Escolaridade } \\
\hline Analfabeto & 13 & 13 & 7 & 7 & 20 & 10 \\
\hline $1^{\circ}$ grau completo & 4 & 4 & 12 & 12 & 16 & 8 \\
\hline $1^{\circ}$ grau incompleto & 70 & 70 & 58 & 58 & 128 & 64 \\
\hline $2^{\circ}$ grau completo & 5 & 5 & 11 & 11 & 16 & 8 \\
\hline $2^{\circ}$ grau incompleto & 4 & 4 & 5 & 5 & 9 & 4,5 \\
\hline Universitário & 3 & 3 & 4 & 4 & 7 & 3,5 \\
\hline Universitário incompleto & 1 & 1 & 3 & 3 & 4 & 2 \\
\hline \multicolumn{7}{|l|}{ Ocupação } \\
\hline Nível I & 57 & 57 & 50 & 50 & 107 & 53,5 \\
\hline Nível II & 33 & 33 & 24 & 24 & 57 & 28,5 \\
\hline Nível III & 3 & 3 & 10 & 10 & 13 & 6,5 \\
\hline Nível IV & 7 & 7 & 16 & 16 & 23 & 11,5 \\
\hline \multicolumn{7}{|l|}{ Renda (salários mínimos) } \\
\hline$<5$ & 67 & $67^{*}$ & 39 & 39 & 106 & 53,0 \\
\hline $5-10$ & 23 & 23 & 34 & 34 & 57 & 28,5 \\
\hline $10-20$ & 9 & 9 & 31 & 31 & 40 & 20,0 \\
\hline$>20$ & 1 & 1 & 6 & 6 & 4 & 3,5 \\
\hline \multicolumn{7}{|l|}{ Categoria } \\
\hline Comunidade USP e Butantã & 16 & 16 & 77 & 77 & 93 & 46,5 \\
\hline Comunidade não USP & 84 & $84^{*}$ & 23 & 23 & 107 & 53,5 \\
\hline \multicolumn{7}{|l|}{ Pressão (mm Hg, média+DP) } \\
\hline sistólica & \multirow{2}{*}{\multicolumn{2}{|c|}{$\begin{array}{l}174+27,4^{*} \\
109+18,1^{*}\end{array}$}} & \multirow{2}{*}{\multicolumn{2}{|c|}{$\begin{array}{c}143+20 \\
88+12\end{array}$}} & & \\
\hline diastólica & & & & & & \\
\hline \multicolumn{7}{|l|}{ Índice de massa corpórea } \\
\hline$(\mathrm{kg} / \mathrm{m} 2)$ & \multicolumn{2}{|c|}{$\cdots$} & \multicolumn{2}{|c|}{$26+6,9$} & & \\
\hline \multicolumn{7}{|l|}{ Hábitos de vida } \\
\hline Fumo & 18 & 18 & 13 & 13 & 31 & 15,5 \\
\hline Atividade física & 19 & 19 & 58 & 58 & 77 & 38,5 \\
\hline Uso hormônio anticoncepcional & 1 & 3,2 & 10 & $16,1^{*}$ & 12 & 9,7 \\
\hline Bebida alcoólica & 47 & 47 & 42 & 42 & 89 & 44,5 \\
\hline Gramas etanol/dia (média+DP) & \multicolumn{2}{|c|}{$91,7+9,0^{*}$} & \multicolumn{2}{|c|}{$16,7+7,0$} & \multicolumn{2}{|c|}{$54,2+8,0$} \\
\hline
\end{tabular}

${ }^{*} p<0,05$

Nível I: Ocupação manual não especializada, Nível II: Ocupação manual especializada 
Observou-se também que os pacientes do Pronto-socorro apresentaram nível da pressão arterial significativamente mais elevado, caracterizando hipertensão arterial de grau moderado a grave $(174 \pm 27,4 / 109 \pm 18,1 \mathrm{~mm}$ Hg vs. $143 \pm 20 / 88 \pm 12 \mathrm{~mm} \mathrm{Hg}, \mathrm{p}<0,05)$.

Em relação aos hábitos de vida, os pacientes do Pronto-socorro referiram ingestão de bebida significativamente mais elevada do que os pacientes do Ambulatório (91,7 $\mathrm{g}$ $\pm 9,0$ vs. 16,7 $\mathrm{g} \pm 7,0$ gramas de etanol/dia, $\mathrm{p}<0,05)$. Apesar do pequeno número de mulheres que citaram fazer uso de hormônio anticoncepcional, a quantidade de pacientes hipertensas acompanhadas no Ambulatório referiram uso significativamente mais elevado $(\mathrm{p}<0,05)$ do que o número de pacientes do Pronto-socorro (10 vs. 2).

Todos os pacientes entrevistados sabiam ter pressão alta e na maioria o tempo de doença foi acima de 5 anos. O motivo mais apontado que levou os hipertensos a descobrirem a pressão alta foi sentir-se mal, com diferença significativa para os pacientes do Pronto-socorro (60\% vs. 45\%, p <0,05). A grande maioria dos pacientes entrevistados afirmou ter o hábito de medir a pressão e em intervalos menores que 6 meses. Os pacientes do Ambulatório referiram medir de forma mais freqüente $(96 \% v s .78 \%, \mathrm{p}<0,05)$ e no momento da consulta médica ( $65 \%$ vs. $20 \%$, $\mathrm{p}<0,05$ ) (Tabela 2).

Tabela 2 - Dados sobre a doença pressão alta e hábito de medir a pressão entre os pacientes hipertensos atendidos no Pronto-socorro e Ambulatório (São Paulo, 2001)

\begin{tabular}{|c|c|c|c|c|c|c|}
\hline \multirow[t]{2}{*}{ Dados sobre a doença } & \multicolumn{2}{|c|}{$\begin{array}{l}\text { Pronto- } \\
\text { socorro }\end{array}$} & \multicolumn{2}{|c|}{ Ambulatório } & \multicolumn{2}{|c|}{ Total } \\
\hline & $\mathbf{n}^{\mathbf{o}}$ & $\%$ & $\mathbf{n}^{\mathbf{o}}$ & $\%$ & $\mathbf{n}^{\mathbf{o}}$ & $\%$ \\
\hline Sabe ter pressão alta & 100 & 100 & 100 & 100 & 200 & 100 \\
\hline \multicolumn{7}{|l|}{ Tempo de pressão alta } \\
\hline$<1$ ano & 15 & 15,0 & 5 & 5,0 & 20 & 10,0 \\
\hline $1-5$ anos & 35 & 35,0 & 28 & 28,0 & 63 & 31,5 \\
\hline $6-10$ anos & 15 & 15,0 & 23 & 23,0 & 38 & 19,0 \\
\hline$>10$ anos & 35 & 35,0 & 44 & 44,0 & 79 & 39,5 \\
\hline \multicolumn{7}{|l|}{ Como descobriu a pressão alta } \\
\hline sentiu-se mal & 60 & $60,0^{*}$ & 45 & 45,0 & 105 & 52,5 \\
\hline mediu em campanha & - & - & 2 & 2,0 & 2 & 1,0 \\
\hline consulta de rotina & 27 & 27,0 & 39 & 39,0 & 66 & 33,0 \\
\hline gravidez & 4 & 4,0 & 11 & 11,0 & 15 & 7,5 \\
\hline mediu pressão em PS & 9 & 9,0 & 3 & 3,0 & 12 & 6,0 \\
\hline Hábito de medir pressão & 78 & 78,0 & 96 & $96,0^{*}$ & 174 & 87,0 \\
\hline \multicolumn{7}{|l|}{ Onde mede a pressão } \\
\hline Consulta médica & 16 & 20,5 & 62 & $64,6^{*}$ & 78 & 44,9 \\
\hline Posto de Saúde & 30 & 38,5 & 14 & 14,6 & 44 & 25,3 \\
\hline Farmácia & 20 & 25,6 & 7 & 7,3 & 27 & 15,5 \\
\hline Em casa & 12 & 15,4 & 13 & 13,5 & 25 & 14,3 \\
\hline \multicolumn{7}{|l|}{ Intervalo mede a pressão } \\
\hline$<6$ meses & 72 & 92,3 & 95 & 99,0 & 167 & 96,0 \\
\hline 6 meses - 1 ano & 5 & 6,4 & 1 & 1,0 & 6 & 3,4 \\
\hline$>1$ ano & 1 & 1,3 & - & - & 1 & 0,6 \\
\hline
\end{tabular}

Outro dado evidenciado no presente estudo foi que os hipertensos do Prontosocorro referiram de modo significativamente mais elevado $(\mathrm{p}<0,05)$ a ocorrência de outros problemas de saúde quando comparados aos do Ambulatório, tais como: diabetes
(42,3\% vs. $23,7 \%)$, problemas cardíacos $(19,2 \%$ vs. $1,7 \%)$ e hipertireoidismo $(11,5 \%$ vs. $0 \%)$. Os pacientes do Ambulatório se diferenciaram apenas na presença de dislipidemia $(40,7 \%$ vs. $15,4 \%, \mathrm{p}<0,05)$, conforme apresentado naTabela 3.
Comparação dos perfis dos pacientes hipertensos atendidos em Pronto-socorro e em tratamento ambulatorial 
Cristiane Garcia Sanchez Angela Maria G. Pierin Décio Mion Jr.

Tabela 3 - Ocorrência de outros problemas de saúde dos pacientes hipertensos atendidos no Pronto-socorro e Ambulatório (São Paulo, 2001)

\begin{tabular}{|c|c|c|c|c|c|c|}
\hline \multirow[t]{2}{*}{ Problemas de saúde } & \multicolumn{2}{|c|}{$\begin{array}{l}\text { Pronto- } \\
\text { socorro }\end{array}$} & \multicolumn{2}{|c|}{ Ambulatório } & \multicolumn{2}{|c|}{ Total } \\
\hline & $\mathbf{n}^{\mathbf{o}}$ & $\%$ & $n^{\circ}$ & $\%$ & $\mathbf{n}^{\circ}$ & $\%$ \\
\hline Sim & 78 & $\mathbf{7 8 , 0}$ & 59 & 59,0 & 137 & 68,5 \\
\hline Diabetes & 33 & $42,3^{*}$ & 14 & 23,7 & 47 & 34,3 \\
\hline Dislipidemias & 12 & 15,4 & 24 & $40,7^{*}$ & 36 & 26,3 \\
\hline Problema osteomuscular & 16 & 20,5 & 19 & 32,2 & 35 & 25,5 \\
\hline Problemas cardíacos & 15 & $19,2^{*}$ & 1 & 1,7 & 16 & 11,7 \\
\hline Insuficiência renal & 13 & 16,7 & 3 & 5,1 & 16 & 11,7 \\
\hline Enxaqueca & 5 & 6,4 & 7 & 11,9 & 12 & 8,8 \\
\hline Hipertireoidismo & 9 & $11,5^{*}$ & - & - & 9 & 6,6 \\
\hline Doenças respiratórias & 7 & 9,0 & 2 & 3,4 & 9 & 6,6 \\
\hline Obesidade & 1 & 1,3 & 3 & 5,1 & 4 & 3,0 \\
\hline Outros & 2 & 2,6 & 3 & 5,1 & 5 & 3,6 \\
\hline
\end{tabular}

Os pacientes do Pronto-socorro referiram deixar de tomar o remédio mais que os pacientes do Ambulatório (35\% vs. 19\%, p < 0,05). Os motivos mais apontados foram o custo das medicações $(85 \%)$, esquecimento $(24 \%)$ e efeitos indesejáveis (24\%) (Tabela 4).

Tabela 4 - Motivos apontados pelos pacientes hipertensos atendidos no Pronto Socorro e no Ambulatório para deixarem de tomar remédio (São Paulo, 2001)

\begin{tabular}{|c|c|c|c|c|c|c|}
\hline \multirow[t]{2}{*}{$\begin{array}{l}\text { Deixou de tomar os } \\
\text { remédios/motivos }\end{array}$} & \multicolumn{2}{|c|}{$\begin{array}{l}\text { Pronto- } \\
\text { socorro }\end{array}$} & \multicolumn{2}{|c|}{ Ambulatório } & \multicolumn{2}{|c|}{ Total } \\
\hline & $\mathbf{n}^{\mathbf{o}}$ & $\%$ & $\mathbf{n}^{\mathbf{o}}$ & $\%$ & $\mathbf{n}^{\mathbf{o}}$ & $\%$ \\
\hline Sim & 35 & $35^{*}$ & 19 & 19 & 54 & 33 \\
\hline Preço do remédio/ falta dinheiro & 29 & 82,8 & 17 & 89,5 & 46 & 85,2 \\
\hline Esquecimento & 10 & 28,6 & 3 & 15,8 & 13 & 24,1 \\
\hline Efeitos indesejáveis do remédio & 10 & 28,6 & 3 & 15,8 & 13 & 24,1 \\
\hline Achou que estava curado & 8 & 22,8 & 4 & 21,1 & 12 & 22,2 \\
\hline Estava com a pressão baixa & 8 & 22,9 & 4 & 21,1 & 12 & 22,2 \\
\hline Achou que a PA estava boa & 9 & 25,7 & - & - & 9 & 16,7 \\
\hline $\begin{array}{l}\text { Desconhecia que tinha que tomar } \\
\text { droga continuamente }\end{array}$ & 4 & 11,4 & 2 & 10,5 & 6 & 11,1 \\
\hline Só toma quando se sente mal & 2 & 5,7 & - & - & 2 & 3,7 \\
\hline
\end{tabular}

Apenas 35\% dos hipertensos do Ambulatório estavam com a pressão arterial controlada, abaixo de 140/90 mm Hg. Os demais apresentaram hipertensão leve (31\%), moderada $(14 \%)$, grave $(7 \%)$ e hipertensão sistólica isolada (13\%). A maioria dos hipertensos admitidos no Pronto-socorro apresentou níveis de hipertensão grave (43\%) e moderada $(30 \%)$, com redução significante $(\mathrm{p}<0,05)$ na alta (Figura 1).

\section{Ambulatório}

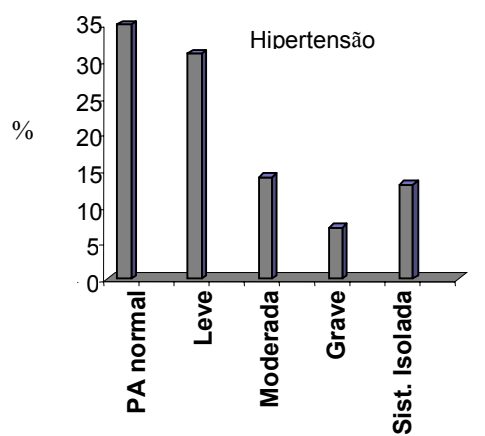

\section{Pronto-socorro}

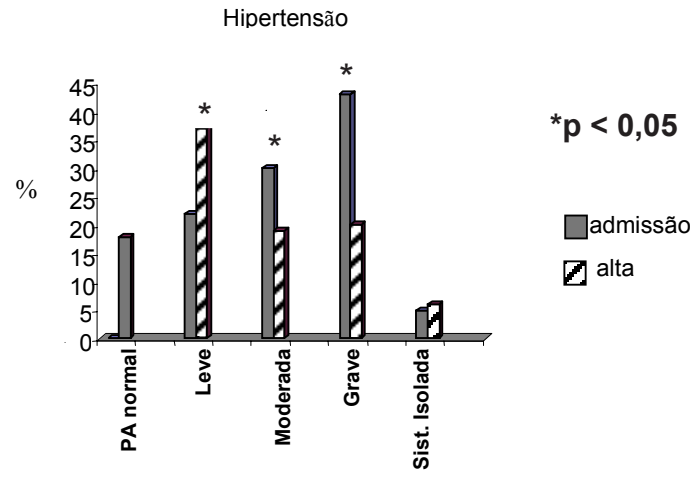

Rev Esc Enferm USP 2004; 38(1): 90-8.
Figura 1 - Níveis da pressão arterial no Ambulatório e Pronto-socorro 
Os anti-hipertensivos em uso mais citados pelos hipertensos considerando os dois grupos, foram os diuréticos (48\%), em monoterapia ou em associação, seguidos dos inibidores da enzima de conversão (30\%), inibidores adrenérgicos (28\%) e antagonistas de canais de cálcio (24\%). O tratamento com um medicamento anti-hipertensivo foi referido por $53 \%$ dos pacientes, com duas ou mais drogas por $21 \%$, não referiram por $8 \%$ e não estavam usando anti-hipertensivos por $18 \%$.
A grande maioria (86\%) dos hipertensos estudados referiu fazer uso de tratamento não medicamentoso. Os pacientes do Ambulatório apontaram redução de gorduras na dieta e realização de exercícios físicos de modo significativamente mais elevado do que os pacientes do Pronto-socorro (93\% vs. 65\% e $47 \%$ vs. $2 \%, \mathrm{p}<0,05)$, as demais medidas apontadas foram: redução do sal (99\%), de peso $18 \%)$, de fumo (3\%), de estresse $(2 \%)$ e de ingestão de bebida alcoólica (2\%) (Tabela 5).

Tabela 5 - Tratamento não farmacológico citado pelos pacientes hipertensos do Pronto-socorro e Ambulatório (São Paulo, 2001)

\begin{tabular}{|c|c|c|c|c|c|c|}
\hline \multirow[t]{2}{*}{ Tratamento não farmacológico } & \multicolumn{2}{|c|}{$\begin{array}{l}\text { Pronto- } \\
\text { socorro }\end{array}$} & \multicolumn{2}{|c|}{ Ambulatório } & \multicolumn{2}{|c|}{ Total } \\
\hline & $\mathbf{n}^{\mathbf{o}}$ & $\%$ & $\mathbf{n}^{\mathbf{o}}$ & $\%$ & $\mathbf{n}^{\mathbf{o}}$ & $\%$ \\
\hline Sim & 82 & 82,0 & 90 & 90,0 & 172 & 86,0 \\
\hline Redução do sal & 80 & 97,5 & 90 & 100,0 & 170 & 98,8 \\
\hline Redução de gordura & 53 & 64,6 & 84 & $93,3^{*}$ & 137 & 79,6 \\
\hline Exercício físico & 2 & 2,4 & 42 & $46,6^{*}$ & 44 & 25,6 \\
\hline Redução do peso & - & - & 31 & 34,4 & 31 & 18,0 \\
\hline Uso de chás & 9 & 11,0 & 5 & 5,5 & 14 & 8,1 \\
\hline Redução do fumo & 1 & 1,2 & 5 & 5,5 & 6 & 3,5 \\
\hline Redução do estresse & 1 & 1,2 & 3 & 3,3 & 4 & 2,3 \\
\hline Redução de bebida alcoólica & 1 & 1,2 & 3 & 3,3 & 4 & 2,3 \\
\hline Outros & 3 & 3,7 & 2 & 2,2 & 5 & 2,9 \\
\hline
\end{tabular}

Os hipertensos do Pronto-socorro, no momento da alta, apresentaram pressão arterial mais baixa do que na admissão $(174 \pm$ $27 / 109 \pm 18$ vs $153 \pm 30 / 95 \pm 17, \mathrm{p}<0,05), \mathrm{e}$ o tratamento medicamentoso no atendimento do Pronto-socorro foi monoterápico, prevalecendo o uso de Inibidores da enzima de conversão (67\%), seguido à distância pelos antagonistas de canais de cálcio $(10 \%)$ e diuréticos (10\%).

Os dados da Tabela 6 demonstram que o conhecimento sobre a doença e o tratamento não foi diferente entre os hipertensos do Prontosocorro e Ambulatório, além de se verificar altos índices de conhecimento nos itens analisados.
Comparação dos perfis dos pacientes hipertensos atendidos em Pronto-socorro e em tratamento ambulatorial

Tabela 6 - Conhecimento sobre a doença dos pacientes hipertensos atendidos no Pronto-socorro e no Ambulatório (São Paulo, 2001)

\begin{tabular}{|c|c|c|c|c|c|c|}
\hline \multirow{2}{*}{$\begin{array}{c}\text { Conhecimento sobre a Hipertensão } \\
\text { Arterial }\end{array}$} & \multicolumn{2}{|c|}{$\begin{array}{l}\text { Pronto- } \\
\text { socorro }\end{array}$} & \multicolumn{2}{|c|}{ Ambulatório } & \multicolumn{2}{|c|}{ Total } \\
\hline & $\mathbf{n}^{\mathbf{o}}$ & $\%$ & $\mathbf{n}^{\mathbf{o}}$ & $\%$ & $\mathbf{n}^{\mathbf{o}}$ & $\%$ \\
\hline Pressão alta é doença grave & 86 & 86,0 & 76 & 76,0 & 162 & 81,0 \\
\hline Pressão alta não tem sintoma & 42 & 42,0 & 41 & 41,0 & 83 & 41,5 \\
\hline $\begin{array}{l}\text { A pressão é alta quando está maior que } \\
140 / 90 \mathrm{~mm} \mathrm{Hg}\end{array}$ & 77 & 77,0 & 79 & 79,0 & 156 & 78,0 \\
\hline Pressão alta traz complicações & 94 & 94,0 & 95 & 95,0 & 189 & 94,5 \\
\hline $\begin{array}{l}\text { Tratamento para pressão alta é para toda } \\
\text { vida }\end{array}$ & 91 & 91,0 & 88 & 88,0 & 179 & 89,5 \\
\hline Pressão alta pode ser tratada sem remédio & 30 & 30,0 & 42 & 42,0 & 72 & 36,0 \\
\hline Exercício físico ajuda controlar pressão & 77 & 77,0 & 85 & 85,0 & 162 & 81,0 \\
\hline Reduzir o peso ajuda controlar a pressão & 88 & 88,0 & 94 & 94,0 & 182 & 91,0 \\
\hline Reduzir o sal ajuda a controlar a pressão & 99 & 99,0 & 99 & 99,0 & 198 & 99,0 \\
\hline Estresse pode causar pressão alta & 100 & 100 & 99 & 99,0 & 199 & 99,5 \\
\hline
\end{tabular}


Cristiane Garcia Sanchez Angela Maria G. Pierin Décio Mion Jr.
As variáveis que foram estatisticamente significativas na análise univariada, foram submetidas a um modelo de regressão logística. A análise multivariada revelou que apenas a baixa renda, o local onde mede a pressão e o fato de deixar de tomar o remédio exerceram diferenças fortemente significativas $(\mathrm{p}<0,05)$ entre os pacientes atendidos no Pronto-socorro e os atendidos no Ambulatório (Tabela 7).

Tabela 7 - Variáveis utilizadas no modelo de regressão logística que exerceram diferenças significativas entre os grupos do Pronto-socorro e do Ambulatório (São Paulo, 2001)

\begin{tabular}{lccc}
\hline \multicolumn{1}{c}{ Variáveis } & Valor de p & "odds ratio" & $\begin{array}{c}\text { Intervalo de } \\
\text { confiança }\end{array}$ \\
\hline Renda & 0,0001 & 2,837 & $1,728-4,660$ \\
Onde mede a pressão & 0,0009 & 2,236 & $1,389-3,599$ \\
Deixou de tomar remédio & 0,0349 & 3,134 & $1,084-9,057$ \\
\hline
\end{tabular}

\section{DISCUSSÃO}

Os principais achados do presente estudo evidenciaram perfil desfavorável dos pacientes hipertensos atendidos no Prontosocorro, quando comparados aos pacientes em seguimento ambulatorial. Dentre as características biossociais, a menor renda salarial e o fato de não pertencer à comunidade Universidade de São Paulo e Butantã podem contribuir para a não realização adequada do tratamento para a hipertensão arterial e dificultar o acesso aos serviços de saúde. Desvantagens sociais e precárias condições de vida acompanham-se, de modo geral, de um maior risco de adoecer e de morrer, por qualquer causa, em todas as idades, em ambos os sexos ${ }^{(13-14)}$.

Associada às características biossociais desfavoráveis, também se destacou para os hipertensos do Pronto-socorro o maior consumo de bebida alcoólica. O tipo de bebida alcoólica tem pouca importância, porque o conteúdo de etanol por dia é o principal determinante do efeito hipertensivo, além de se relacionar com as causas de resistência à terapêutica anti-hipertensiva ${ }^{(15-17)}$.

O fato dos pacientes do Pronto-socorro terem descoberto a hipertensão por sentiremse mal mostra a possibilidade do agravamento da doença pela falta de acompanhamento regular. Como o sistema de saúde não provê condições adequadas para o tratamento das doenças crônicas, a procura pelo Prontosocorro torna-se um "atendimento regular" semelhante ao atendimento ambulatorial. Considerando que a hipertensão arterial é uma doença na maioria das vezes assintomática, é importante fator de risco para as doenças cardiovasculares, que mais matam no mundo, e compreendida como um problema de saúde pública, faz-se necessário a adoção de ações para o seu controle, sendo prioritário o diagnóstico precoce e o tratamento adequado dos pacientes que buscam os serviços de saúde.

Frente aos resultados do presente estudo é interessante apontar que apesar da grande maioria dos pacientes não ter a pressão arterial controlada, eles referiram medir a pressão com freqüência relativamente satisfatória, o que possibilita diagnóstico da hipertensão. $\mathrm{O}$ fato dos pacientes já terem conhecimento da doença indica que realmente não fazem tratamento adequado, justificando o não controle e a procura pelo atendimento em Pronto-socorro.

Nos últimos anos vem aumentando a freqüência de pessoas que mesmo tendo conhecimento da sua doença hipertensiva, não modificam sua atitude diante da importância do tratamento ${ }^{(13)}$. Convencer um paciente, muitas vezes assintomático, de que ele é doente, especialmente quando isto implica em mudanças no estilo de vida ou ainda na obrigação de usar medicamentos para sempre, é um objetivo menos facilmente alcançado. Os profissionais não podem subestimar as dificuldades encontradas na adoção de medidas que alteram substantivamente os hábitos de vida dos pacientes. Nesse sentido, é importante uma boa relação entre a equipe de saúde para uma orientação personalizada, adequando-a aos hábitos de vida e ao poder aquisitivo do paciente.

Considerando que o tratamento farmacológico traz conseqüências para a vida do 
doente e, especialmente, mantém a doença sob controle, a análise dos resultados neste estudo mostrou que a grande maioria dos pacientes apontou que fazia uso de algum tipo de medicação anti-hipertensiva. Porém, quase um terço dos pacientes entrevistados referiu algum motivo para deixar de tomar o remédio nas duas semanas que precederam a coleta de dados.

A falta de adesão ao tratamento medicamentoso tem chegado a aproximadamente $40 \%$ dos pacientes com hipertensão, que pode ser atribuída a vários fatores, incluindo custo da medicação, informação inadequada do paciente, uso de mais de uma droga, efeitos adversos, cronicidade da doença e ausência de sintomas específicos ${ }^{(18-20)}$.

Desde que foi estabelecido que o tratamento da hipertensão arterial é eficaz, seria esperado que a maioria dos pacientes hipertensos estivesse se beneficiando destes conhecimentos. No entanto, em grande escala isto ainda não é verificado face aos inúmeros fatores intervenientes tornando mais difícil o controle da hipertensão, principalmente nas classes sociais menos favorecidas, o que pode acarretar maior demanda nos serviços de urgência. A maioria das hospitalizações, urgências, emergências e mortes seria evitável caso houvesse decisão dos políticos de saúde para assumirem com seriedade a hipertensão arterial como problema prioritário de saúde pública $^{(13)}$.

Um aspecto positivo a ser destacado foi que os hipertensos entrevistados indicaram uso de medidas não medicamentosas para o tratamento da hipertensão, como controle do sal, redução do peso corporal, redução na ingestão de gorduras e realização de exercício físico. O tratamento não medicamentoso, baseado em medidas que visam modificações no estilo de vida, reduz significativamente a pressão arterial e deve ser indicado a todos os hipertensos, além de atuarem favoravelmente sobre outros fatores de riscos cardiovasculares, mudando a incidência de doença

\section{REFERÊNCIAS}

(1) Zampaglione B, Pascale C, Marchisio M, Cavallo-Perin P. Hypertensive urgencies and emergencies. Prevalence and clinical presentation. Hypertension 1996; 27(1):144-7. cardíaca, vascular e renal ${ }^{(21-23)}$. Neste contexto, destaca-se a importância do papel da equipe multiprofissional no sentido de reunir esforços para possibilitar uma assistência que atenda as reais necessidades dos hipertensos.

A avaliação do conhecimento sobre hipertensão arterial e tratamento mostraram índices elevados e sem diferenças significativas entre os pacientes atendidos no Pronto-socorro e aqueles em tratamento ambulatorial, indicando que o conhecimento não foi fator discriminante e que o problema é bem mais complexo, com múltiplas influências, que vão desde as características biopsicos-sociais dos pacientes, fatores relativos ao tratamento, relacionamento com equipe de saúde, até as políticas de saúde vigentes. No momento tanto no âmbito federal e estadual, estão sendo direcionados esforços no sentido de estabelecer propostas que viabilizem assistência adequada às pessoas hipertensas.

\section{CONCLUSÃO}

Concluindo, os resultados do atual estudo evidenciaram o perfil desfavorável dos hipertensos atendidos em situação de emergência, quando comparados com aqueles em seguimento ambulatorial o que pode maximizar ainda mais a dificuldade no controle da hipertensão arterial. As diferenças significativas entre os dois grupos estudados como renda mais baixa, é um fator que dificulta ou até impossibilita o tratamento medicamentoso, mostrando uma baixa adesão ao tratamento. Além disso, não ter acesso aos serviços para seguimento regular reforça mais uma vez a necessidade de medidas que visam o conhecimento das características dos pacientes hipertensos atendidos tanto em Pronto-socorro como dos seguidos regularmente no Ambulatório. Estes problemas devem ser enfrentados pela Instituição e pela equipe de saúde, assegurando que os hipertensos recebam os benefícios do tratamento para o efetivo controle da pressão arterial.
Comparação dos perfis dos pacientes hipertensos atendidos em Pronto-socorro e em tratamento ambulatorial 
Cristiane Garcia Sanchez Angela Maria G. Pierin Décio Mion Jr.
(3) Zavarella MS. Understanding \& managing hypertensive emergencies. J Emerg Med Serv 2000; 25:50-61.

(4)Vaughan CJ, Delanty N. Hypertensive emergencies. Lancet 2000; 356:411-7.

(5) Karras DJ, Wald DA, Harrigan RA. Elevated blood pressure in an Urban Emergency Department: prevalence and patient characteristics. Acad Emerg Med 2001; 8:559.

(6) $3^{\circ}$ Consenso Brasileiro de Hipertensão Arterial, 1998; Campos do Jordão (SP). [on line]. Campos do Jordão: Sociedade Brasileira de Hipertensão, Cardiologia e Nefrologia, 1998. Disponível em <http://departamentos.cardiol.br/ c b h a / p u b li c a ç õ e s/ c o n s e n s o 4 / apresentação.asp> (12 dez 2001).

(7) The Six Report of the Joint National Committee on Prevention, Detection. Evaluation and treatment of high blood pressure. Arch Int Med 1997; 157:2413-45.

(8) World Health Organization. International Society of Hypertension Guidelines for the Management of Hypertension. J Hypertens 1999; 17:151-83.

(9) Lotufo PA. As doenças cardiovasculares no Brasil: estudo de caso da tendência da mortalidade no Estado de São Paulo 1970-1989. [dissertação] São Paulo (SP): Faculdade de Saúde Pública da USP; 1993.

(10) Busnello RG, Melchior R, Faccin C, Vettori D, Petter J, Moreira LB et al. Características associadas ao abandono do acompanhamento de pacientes hipertensos atendidos em um ambulatório de referência. Arq Bras Cardiol 2001; 76(5):352-4.

(11) Pierin AMG, Mion Jr D. Adesão ao tratamento anti-hipertensivo - conceito, avaliação e fatores determinantes. Hipertensão 1999; 2:9-12.

(12) Lahdenpera TS, Kyngas HA. Levels of compliance shown by hypertensive patients their attitude toward their illness. J Adv Nurs 2001;34:189-95.

(13) Lessa I. Não adesão ao tratamento da hipertensão: conseqüências socioeconômicas para o indivíduo e para a sociedade. In: Nobre F, Mion Jr D. Adesão ao tratamento: o grande desafio da hipertensão. São Paulo: Lemos; 2001. p. 96.

(14)Thomas F, Rudnichi H, Bacri AM, Bean K, Guize L, Benetos A. Cardiovascular mortality in hypertensive men according to presence of associated risck factors. Hypertension 2001; 37(5):1256-61.
(15) Arkwight PD, Beilin LJ. Effects of alcohol use and other aspects of lifestyle on blood pressure levels and prevalence of hypertension in a working population. Circulation 1994; 20:60-6.

(16) Hillbom M, Nummien H, Juvela S. Recent heavy drinking of alcohol embolic stroke. Stroke 1999; 30(11)2307-12.

(17) Oparil S, Calhoun DA. Managing the patient with hard-to-control hypertension. Am Fam Phisician 1998; 57(5):1007-14.

(18) Pierin AMG. Adesão ao tratamento: conceitos. In: Nobre F, Mion Junior D. Adesão ao tratamento: o grande desafio da hipertensão. São Paulo: Lemos; 2001, cap 1, p.25. 2001.

(19) Krasilcic S. Índices de adesão aos tratamentos medicamentoso e não medicamentoso no Brasil e no mundo.In: Nobre F, Mion Junior D. Adesão ao tratamento: o grande desafio da hipertensão. São Paulo: Lemos; 2001. p.47.

(20) Enlund H, Jokisalo E, Wallenius S, Korhonen M. Patient-perceived problems, compliance, and the outcome of hypertension treatment Pharm World Sci 2001; 23(2): 60-4

(21) Cutler JA, Follmann D, Allender PS. Randomized trials of sodium reduction: an overview. Am J Clin Nutr 1997; 652 (Suppl): 643S- 51S.

(22) Intersalt Cooperative Research Group. Intersalt: an international study of electrolyte excretion and blood pressure. Results for 24 hour urinary sodium and potassium excretion. BMJ 1988; 297(6644):319-28.

(23) Negrão CE, Tinucci T, Rondon MUPB. Sedentarismo e fatores de risco de doenças cardiovasculares. Hipertensão 1999; 2:23-9. 